\title{
Chapter 9 \\ Optical waveguide-based biosensor for label-free monitoring of living cells
}

\author{
N. Orgovan ${ }^{1,2}$, B. Szabó ${ }^{1,2}$ and R. Horvath ${ }^{1}$
}

\begin{abstract}
Here, we briefly discuss the past, present, and possible future of label-free optical biosensors in cell research, especially focusing on the kinetic monitoring of cellular adhesion. Currently available optical biosensors possess outstanding potentials still not rightfully recognized and still waiting to be fully exploited in the field of cell science. Thus, during the description we give special emphasis to the advantages that the state-of-the-art optical cell-based biosensors possess as compared to microscope- or force measurement-based techniques widely used to characterize cell adhesion. To name here only a few, they enable label-free detection close to a planar sensor surface, have high sensitivity, and generate superior quality kinetic data. Such information-rich kinetic data, in turn, can be analyzed in-depth and comparatively. To exemplify the importance of in-depth kinetic analysis, we review a recent study, in which the Epic Ben Top high-throughput optical biosensor was used to measure the dependence of cancer cell adhesion kinetics on the surface density of integrin ligands. Based on the kinetic data, a model enabling the label-free determination of the dissociation constant of the adhesion ligands bound to their native cell membrane receptors has been constructed. Perspective, of the technology is briefly discussed.
\end{abstract}

\subsection{Label-free optical biosensors in cell adhesion research}

Cell adhesion is a fundamental biological process during which a cell anchors itself to a suitable surface and spreads on it, obtaining a well-spread morphology characteristic to the cell type. Cell adhesion plays cardinal roles on the level of individual cells - e.g. in intracellular signaling [1, 2], migration [3], proliferation [4], differentiation [5],

\footnotetext{
${ }^{1}$ Nanobiosensorics Group, Hungarian Academy of Sciences for Energy Research, Institute for Technical Physics and Materials Science, H-1120 Budapest, Hungary

${ }^{2}$ Department of Biological Physics, Eötvös University, H-1117 Budapest, Hungary
} 
gene expression [6], and in general, cell fate determination [7] - as well on the level of multicellular organisms - e.g. in cell-cell communication [8], immune function $[9,10]$, cancer development $[11,12]$, or in the initiation and pathogenesis of bacterial [13] and viral diseases [14]. For decades the phenomenon of cell adhesion has thus been enjoying the ever-increasing scientific interest of various interdisciplinary fields, including materials science, pharmacology, biophysics, and more, both in basic and applied research. This prominent attention has stimulated many technological developments which gave rise to new techniques or modified already existing ones, all to enable a more detailed characterization of cell adhesion. Based on the approach they use, these can be classified into three main groups.

First, a class of techniques attempts to characterize cell adhesion in the most direct manner: by quantifying cell-exerted forces. Some of these techniques measure the adhesion force by actually detaching the cell from its substrate (e.g. flow chamber $[15,16]$, variants of atomic force microscopy [17, 18], or micropipette [19]), while others characterize cell-exerted forces through the cell-caused deformations of an elastic substrate (variants of cell traction force microscopy and their predecessors [20, 21]).

In contrast, another class of techniques focuses on the visualization and/or tracking of the cell and its adhesion-associated subcellular structures (e.g. those of filamentous actin, or focal adhesion components) [22]. Visualization is performed under a microscope (e.g. wide-field, confocal, electron, or total internal reflection microscope), which is subsequently followed by in-depth image analysis [22].

Surface-sensitive label-free optical biosensors constituting the third class of techniques are relative newcomers to cell adhesion science [23]. (Although impedance-based label-free biosensors can also be used to measure cell adhesion, space limitations force us to omit them from this discussion. For references, see $[24,25])$. Several members of the family have been shown to be able to measure cell adhesion, including surface plasmon resonance (SPR) [26, 27], optical waveguide light mode spectroscopy (OWLS) [28-31], photonic crystal (PC) biosensors [32], and resonant waveguide grating (RWG, or more commonly recognized as Epic) biosensors [33, 34]. Albeit they have been on the horizon for a considerable time, and albeit they offer promising potentials to the field, their introduction to cell adhesion research has been long delayed. This was partially due to the aversion that encompassed their unselective detection mechanism [35]; they are sensitive to any process which is accompanied by refractive index (RI) changes in a thin layer closest to the sensor surface [28]. For this, they have often been referred as black boxes, and their signal being obscure and difficult to interpret [35]. However, with an appropriate surface chemistry and rigorous experimental controls in hand, such biosensors can readily and specifically measure the adherency of a population of cells. Indeed, the biosensor signal integrates changes in both the size of the sensor area covered by cells and the optical density therein (i.e. it depends on the density of filamentous actin near the surface, and on the number and size of focal adhesions), which makes the signal an excellent single measure of cell adherency. Further reasons for the delay in permeation of optical biosensorics to cell science have been (i) the lack of high-throughput in the case of the first-generation, and (ii) the high cost in the case of the second-generation instruments (SPR, OWLS, 
and PC, Epic systems, respectively). Accordingly, first-generation platforms were not suitable for cutting-edge cell (adhesion) research, since single measurements conducted hours apart could be hardly compared due to the inter-experimental variability inherent to living cells. In contrast, second-generation systems were microplate-based high-throughput biosensors, but the first of their kind was an expensive platform with integrated robotics, and hence predominantly only some pharmaceutical companies could afford to buy it.

With the recent commercialization of the small-footprint next-generation optical biosensor, the Epic BenchTop (Epic BT) [33, 36] (and with the permeation of the EnSpire benchtop multimodalmicrotiter plate reader, which combines Epic label-free technology with labeling technologies), we expect the long-stand status quo to change soon, i.e. the potentials of label-free biosensorics to be rightfully recognized and exploited in cell science. In particular, Epic BT offers the following set of advantages: it (i) detects RI changes only in an approximately $150 \mathrm{~nm}$ thick layer closest to a planar sensor surface (which is the most relevant in cell adhesion) $[28,36]$; (ii) produces an integrated signal which is an excellent single measure of cell adherency $[31,37]$; (iii) does not require labeling of any kind to monitor cell behavior; (iv) as a microplate-based system, offers high-throughput detection; (v) yields superior quality kinetic data, which can be subjected to in-depth kinetic analysis [34]. This latter feature in itself is such that the microscope- or force measurement-based techniques can hardly compete with. Hecoming increasingly evident that kinetic analysis is the key to a more detailed characterization of the effect of; e.g. substrate modifications or drug treatments on cellular behavior, including cell adhesion. Given their high sensitivity, surface-sensitive evanescent field-based techniques can detect not only large-scale, but also tiny variations during adhesion, thus molecular movements and rearrangements in the basal membrane of the adhered cells can be monitored in real time. These dynamic changes are often termed as dynamic mass redistribution (DMR) in the literature [23, 33, 38-40]. The conceptually different types of DMRs are briefly summarized in Figure 9.1.

The forthcoming years may bring further innovations to commercially available label-free optical biosensors, so they could e.g. (i) measure the adhesion and signaling of single cells; (ii) combine high-throughput detection with multimode waveguides enabling depth profiling of cell RI variations [30]; or (iii) use flow-through microfluidics in a high-throughput arrangement $[38,41]$.

\subsection{The Epic Bench Top optical biosensor}

The Epic BT system (Corning Incorporated, Corning, NY, USA) is an evanescent field-based optical biosensor [42] allowing high-throughput label-free detection at a solid-liquid interface [23, 33, 36, 40]. It accepts 96- or 384-well Society for Biomolecular Screening (SBS) standard format biosensor microplates. The bottom of an Epic microplate serves as a planar optical waveguide - i.e. a thin, high refractive-index, transparent dielectric layer (waveguide layer, made of the biocompatible material niobium pentoxide [43]) on a thicker substratum. At the 

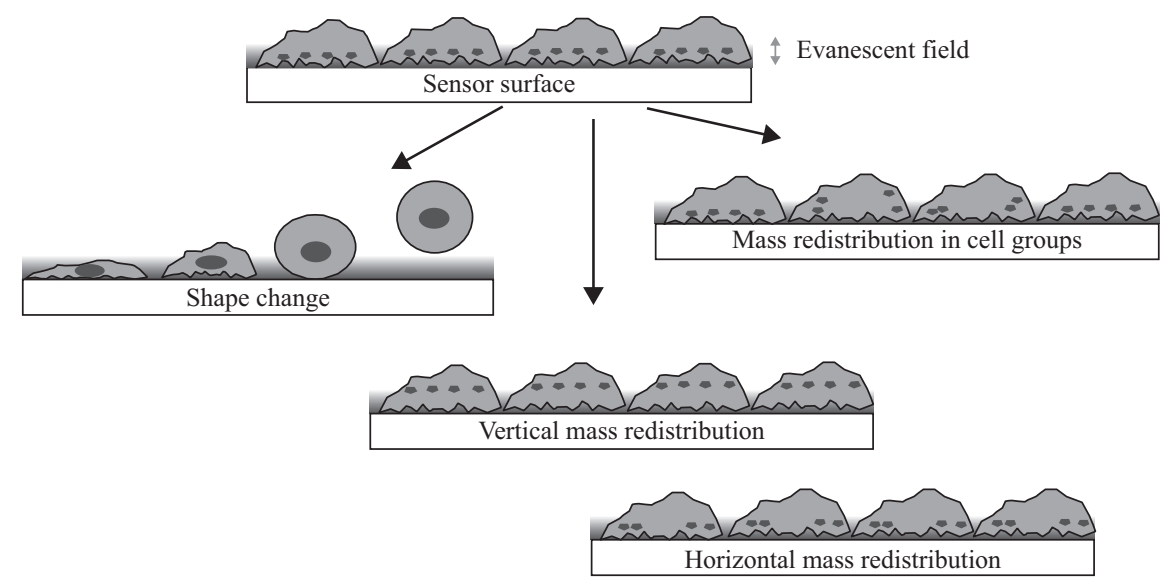

Figure 9.1 Schematic illustration of cell activity detection with label-free optical biosensors. Practically, all types of cellular activities are accompanied by dynamic mass redistributions (DMRs), which in turn generally cause a net change in the local refractive index. A detectable change occurring in the sensing layer of the biosensor (evanescent field, illustrated as a red layer illuminating only the bottom $\sim 150 \mathrm{~nm}$ high portion of cells) provokes a biosensor response (e.g. a spreading curve). Conceptually, four types of mass redistributions can be distinguished. Considerable changes in cell shape involve large-scale DMRs in directions both vertical and horizontal to the planar sensor surface. This is the case during cell spreading when spherical cells enter the sensing layer, which is followed by cell attachment to, and cell spreading on the appropriately prepared sensor surface. In contrast, cells already spread on the sensor surface can exhibit intracellular DMR which is dominant in either the vertical or horizontal direction, while the cell shape does not change considerably. Typically this is the case when spread cells are stimulated or treated with highly specific effector molecules. The detection of horizontal DMR within cells requires a high spatial-resolution biosensor, a kind which is currently not commercially available. Still, biosensors with modest spatial resolution are already successfully used to detect larger scale spatial variances in DMR responses (e.g. when a group of cells respond to a treatment, others not)

position of each well, an optical grating is embedded into the waveguide layer to enable the in coupling of the illuminating light; thus separate biosensors are created. light beams undergo total internal reflections at the inner surfaces of the waveguide layer, and gain a phase shift upon each reflection. The extent of the acquired phase shift depends on the RI of the medium being closest to the reflecting surface (because an exponentially decaying electromagnetic field, 
called an evanescent field, penetrates into a $\sim 150 \mathrm{~nm}$ thick layer of the neighboring medium and probes the local RI [28, 44]). Light beams in coupled by the same grating interfere with each other, but only positive interference results in guiding. This criterion is met only at a certain illuminating wavelength, called the resonant wavelength $(\lambda)$. Any process accompanied by RI-variations in the $50 \mathrm{~nm}$ thick layer over the biosensor surface alters the acquired phase shift

en the beams undergo reflections at the waveguide layer-sample interface. This untunes the resonance but guiding can resume at an illuminating wavelength $\lambda^{\prime} \neq \lambda$. The primary signal output by the Epic BT system is the shift of the resonant wavelength $\left(\Delta \lambda=\lambda^{\prime}-\lambda\right)$ in each well.

In practice, all wells of an Epiemieroplate are simultaneously read out every $3 \mathrm{~s}$ by sweeping the illuminating wavelength through a range of $15000 \mathrm{pm}$ with $0.25 \mathrm{pm}$ precision [42]. The guided wavelength is outcoupled by the same grating used for incoupling, and the resonant wavelength distribution within each well is imaged with a spatial resolution of $\sim 90 \mu \mathrm{m}$ using a complementary metal-oxide semiconductor (CMOS) camera. The small footprint and tolerance to high temperatures of the Epic BT allows it to be placed into a non-humidified cell incubator and, therefore, a better approximation of the in vivo environmental conditions can be provided for the investigated cells.

\subsection{Cell adhesion on tailored surfaces}

In a work described previously, we aimed at measuring and characterizing the dependence of cell adhesion kinetics on the surface density of adhesion ligands in a label-free manner [34]. As such, the work fitted into a hot multidisciplinary research topic concerned with the relative relevance of individual substrate properties in cell behavior determination. Tailoring of a given substrate property either biochemical (e.g. the density, orientation, or variability of adhesion ligands) or physical (e.g. elasticity, hidropout affecting all the others is generally a challenging task. Similarly so is the proper and detailed quantitative characterization of the effects of this modification on cell behavior.

Given the relative ease it can be accomplished with, many have tuned the surface density of adhesion ligands (especially that of the RGD tripeptide) and investigated how the cells respond [45-49]. Various approaches enable the average surface density of the RGD motif (arginine-glycine-aspartic acid)_ a minimal integrin recognition sequence present in several key proteins of the extracellular matrix $[50,51]$ to be tuned at will [45-49]. However, one of the most intriguing recent studies has relied on an advanced technique, called block copolymer micelle nanolithography, to enable the RGD motifs to be positioned in a strict nanoscale order on a planar surface [52]. As it has turned out, the degree of ordering has a substantial impact on cell spreading. Cell attachment and spreading on an ordered nanopattern of ligands were highly restricted when the ligand spacing was increased beyond $\sim 70$ $\mathrm{nm}$. In contrast, randomly distributed ligands with an average interligand distance of more than $92 \mathrm{~nm}$ were still able to promote marked cell spreading [52]. It has been 
claimed that the failure of cell spreading in the former case was due to the overly large interligand distances restricting effective integrin clustering, and the spreading observed in the latter case could be attributed to locally higher ligand densities that are sufficient to promote clustering.

Notwithstanding the impressive work done in the field, it seems like most investigations got stuck at the level of quantifying cell adhesion and spreading at a single time point and, therefore, could only imperfectly describe the effect of substrate modifications (e.g., adhesion-enhancing or -inhibitory). The dynamic aspects of adhesion and spreading have hitherto very rarely been considered [45, 46, 53, 54], mainly because only few techniques enable these processes to be monitored with adequate data quality, especially without the incorporation of labels that may potentially perturb cellular behavior.

\subsection{The dependence of cell adhesion kinetics on the surface density of integrin ligands, as measured with the Epic BT biosensor}

As mentioned earlier, surface-sensitive label-free biosensors are inherently capable of generating good quality kinetic data. Thus, we used Corning's next-generation highthroughput optical biosensor, the Epic BT, to measure the dependence of cell adhesion kinetics on the average surface density of RGD motifs (for the original report, see Reference 34). The protocol and workflow for a typical label-free cell adhesion assay-carried-out utilizing the-high-throughput Epic BT is detailed in a recent book chapter [55].

To tune the average surface density of RGD motifs, we used two copolymers, the biologically inactive PLL-g-PEG (poly(L-lysine)-graft-poly(ethylene glycol)), and its RGD-functionalized counterpart, PLL- $g$-PEG-RGD. If immobilized on a surface, the former functions as a protein-resistant and cell-repellent agent [56], while the latter is selectively recognized by a subgroup of adhesion receptor integrins [57], and thus induces cell adhesion [48]. The copolymers were immobilized on the biosensors (Epic microplate wells) via room-temperature physisorption from coating solutions. Coating solutions were obtained by mixing the stock solutions of PLL-g-PEG and PLL- $g$ PEG-RGD (both dissolved to a final concentration of $1 \mathrm{mg} \mathrm{ml}^{-1}$ ) in the desired ratios. As described earlier [34], the surface density of RGD motifs was tuned by varying the volume percent $Q$ of the PLL- $g$-PEG-RGD solution in the mixed solution of copolymers. The average surface density of RGD ligands $\left(\nu_{\mathrm{RGD}}\right)$, as well as the average RGD-to-RGD distance $\left(d_{\text {RGD-RGD }}\right)$ could be easily calculated using $Q$ and the molecular quantities characterizing the composition of the copolymers [34, 49]. (When discussing trend-like effects of RGD-tuning, $v_{\mathrm{RGD}}$ and $d_{\mathrm{RGD}-\mathrm{RGD}}$ are freely interchanged in further text). In the work described in Reference $34_{2} v_{\mathrm{RGD}}$ was tuned over four orders of magnitude.

Having the surfaces prepared, the microplate wells were given assay buffer (HBSS with $20 \mathrm{mM}$ HEPES). Next, we established a baseline with the biosensor, then introduced the suspension of HeLa cells into the wells. All experiments were 
done in triplicates. After $2 \mathrm{~h}$, the biosensor signals saturated and thus the biosensor experiment was terminated. A single set of obtained signals is shown in Figure 9.2 as points. As it is seen, surfaces with an average interligand distance of $147 \mathrm{~nm}$ were already able to mildly induce cell adhesion (Figure 9.2). Maximum biosensor responses $\left(\Delta \lambda_{\max }\right)$ increased as a response to decreasing the interligand distance until saturation was reached at around $d_{\mathrm{RGD}-\mathrm{RGD}} \approx 10 \mathrm{~nm}$. The saturation is not surprising considering that the diameter of an integrin in the cell membrane is $8-12 \mathrm{~nm}$ [52], thus ligands closer to each other than this cannot be simultaneously bound.

Given the high resolution of the data set, it could be subjected to kinetic analysis. All the obtained spreading curves followed a symmetrical sigmoid shape, which can most easily be described by the logistic (9.1) [58]:

$$
\Delta \lambda(t)=\frac{\Delta \lambda_{\max }}{1+\exp (-r(t-m))} \Omega
$$

where $\Delta \lambda_{\max }$ is the signal value at the maximum (plateau) of the spreading curve, $r$ is the rate constant of spreading, and $m$ is the time at which the ordinate is exactly $\Delta \lambda_{\max } / 2$. We used (9.1) to fit each individual data sequence (and not the averaged

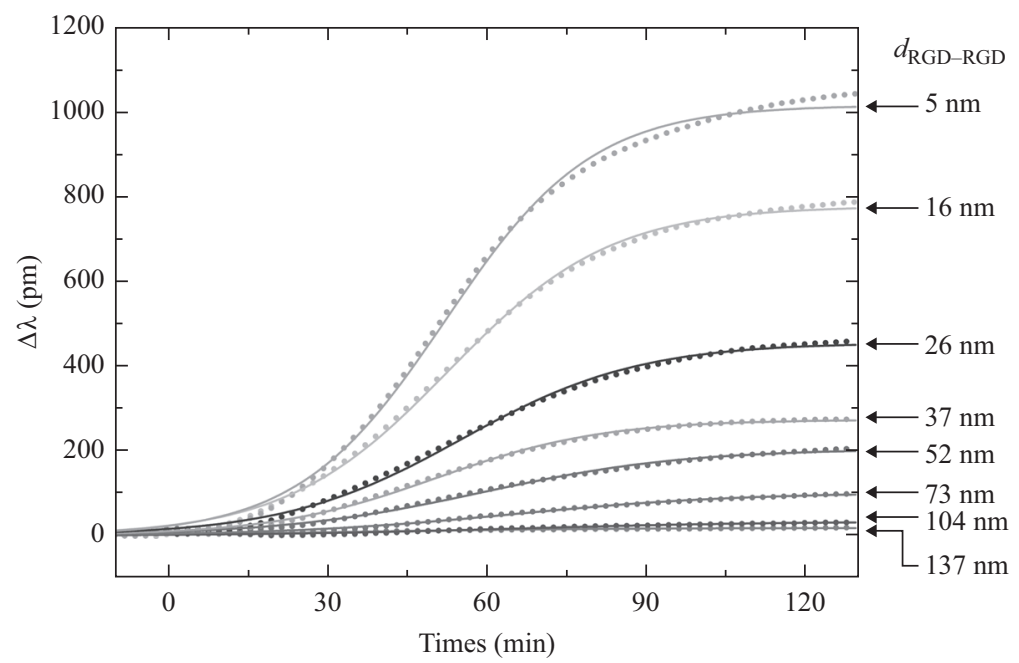

Figure 9.2 Spreading curves (the resonant wavelength shift $\Delta \lambda$ as a function of time) provoked by HeLa cells, as was measured with the Epic BT at different $R G D$ surface densities. The average interligand distances are indicated on the right side of the figure: cells on higher RGD-density surfaces induced higher biosensor signals. Dots: individual spreading curves registered by the Epic BT. Solid curves: fits. Note, that only one series of curves is shown, and some data and the corresponding fits have been omitted from this figure to avoid crowding and overlaps. Figure is replotted from Reference 34 
curves of triplicates, Figure 9.2), then the fitting parameters $\left(\Delta \lambda_{\max }\right.$ and $\left.r\right)$ of triplicates were averaged and their mean values were plotted against $\nu_{\mathrm{RGD}}$ and $d_{\text {RGD-RGD }}$.

$r$ was found to be practically independent of the surface density of RGD motifs (not shown) [34]. This was in accordance with a previous report [46], where substrata were coated with varying amounts of fibronectin, and the rate of contact area increase of isotropically spreading fibroblasts was measured with TIRFM. We have proposed that $r$ most probably depended on the growth of the filopodia governed by actin polymerization and was therefore naturally independent from $v_{\text {RGD }}$ [34].

In contrast to $r$, the maximum biosensor response $\left(\Delta \lambda_{\max }\right)$ did depend on the average surface density of RGD moti $\sigma$ GD) (Figure 9.3) [59]. The backgrounds of this dependency can be understood in light of previous findings $[52,60 \%$ has earlier been shown that if the RGD surface density is decreased, cell acticsion diminishes either abruptly (there is a critical interligand distance) or successively, depending on whether the RGD motifs are positioned in a strict nanoscale order or at random. In our case, the nanoscale distribution of the RGD motifs was disordered, but not completely random: the adsorption of the PLL-g-PEG-RGD molecules corresponded to a random deposition of islands, each with an average of 3 RGD motifs. Still, this dispersion resulted in a highly disordered RGD-distribution $\mathrm{F}_{2}$ and thus the observed successive decrease of cell adhesion with decreasing $v_{\mathrm{RGD}}$ is in nice

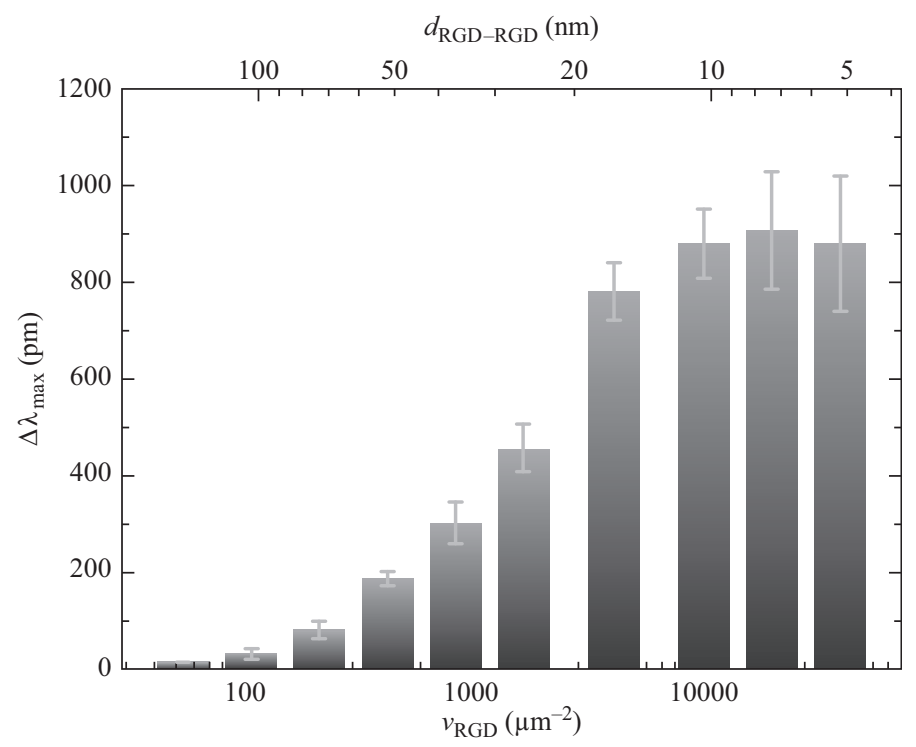

Figure 9.3 Maximum biosensor response $\left(\Delta \lambda_{\max }\right)$ as a function of the average surface density of RGD motifs ( $v_{\mathrm{RGD}}$, bottom axis) and as a function of the average interligand distance ( $d_{\mathrm{RGD}-\mathrm{RGD}}$, top axis). Error bars represent the standard deviation from the mean. Figure is replotted from Reference [34]. 
agreement with expectations [52]. In further accordance with previous findings [52], we obtained the maximum biosensor response at around an average interligand distance of 10 indeed, separation distances smaller than the diameter of an integrin in the cell membrane $(8-12 \mathrm{~nm})$ cannot possibly increase the biosensor response further.

To further analyze the dependence of $\Delta \lambda_{\max }$ on $\nu_{\mathrm{RGD}}$, we assumed that the receptor-ligand interaction can be described as a monovalent binding reaction [34]. Denoting the surface concentrations of the unbound receptor (integrin), unbound ligand (RGD), and that of their bound form as $R, L$ and $B$, respectively, the receptorligand reaction is shown in (9.2):

$$
R+L \underset{k_{\mathrm{d}}}{\stackrel{k_{\mathrm{a}}}{\rightleftharpoons}} B \Omega
$$

According to the kinetic mass action law (KMAL), in equilibrium the attachment and detachment reactions (characterized by two-dimensional rate coefficients $k_{\mathrm{a}}$ and $k_{\mathrm{d}}$, respectively) have equal rates and as show in $(9.3)$ :

$$
B_{\text {eqm }}=\frac{L_{0} R_{0}}{L_{0}+{ }^{2 D} K_{\mathrm{d}}} \curvearrowright
$$

Where $L_{0}=L+B$ and $R_{0}=R+B$ (i.e. $R_{0}=v_{\mathrm{RGD}}$ ), and ${ }^{2 D} K_{\mathrm{d}}=k_{\mathrm{d}} / k_{\mathrm{a}}$ is the two-dimensional dissociation constant. Supposing that $B_{\text {eqm }}$ was directly proportional to the optical response measured at saturation $\left(\Delta \lambda_{\max }\right)$,and fitting the data plotted as $\Delta \lambda_{\max } \sum_{\mathrm{GD}}$ (Figure 9.4) with (Figure-9.3), we found that ${ }^{2 D} K_{\mathrm{d}}=1753 \pm 243 \mu \mathrm{m}^{-2}$.

The relationship between the two- and three-dimensional dissociation constants is given in (9.4):

$$
{ }^{3 D} K_{\mathrm{d}}=\frac{{ }^{2 D} K_{\mathrm{d}}}{l_{\mathrm{c}}} \Omega
$$

where $l_{\mathrm{c}}$ is a characteristic length of the interacting system, often referred to as confinement length $[61,62]$. We proposed $l_{\mathrm{c}}$ to be the average cell-substrate separation distance [34].The extent of separation is the result of the combined effect of nonspecific repulsion and specific bonding forces between the cell and the underlying substrate [61]. Various techniques have been utilized to determine the separation distance, and the obtained average values are typically in the range of 40-160 nm [63-65]. Lacking more precise information, we assumed an average separation distance and an equivalent confinement length of $l_{\mathrm{c}}=100 \mathrm{~nm}$.

Using (9.4), the estimated value of the three-dimensional dissociation constant is ${ }^{3 D} K_{\mathrm{d}} \approx 30 \mu \mathrm{M}$. In comparison, $\alpha I I b \beta 3$ integrins incorporated into a lipid planar bilayer have shown to have an affinity of $1.7 \mu \mathrm{M}$ for an RGD-containing linear peptide (having a very similar amino acid sequence to that used to functionalize the PEG-chains) [66]. The roughly twentyfold discrepancy between this value and ours can be attributed to differences between the investigated systems. First, the platelet integrin $\alpha I I b \beta 3$ is unlikely to have the exact same affinity for the same linear 


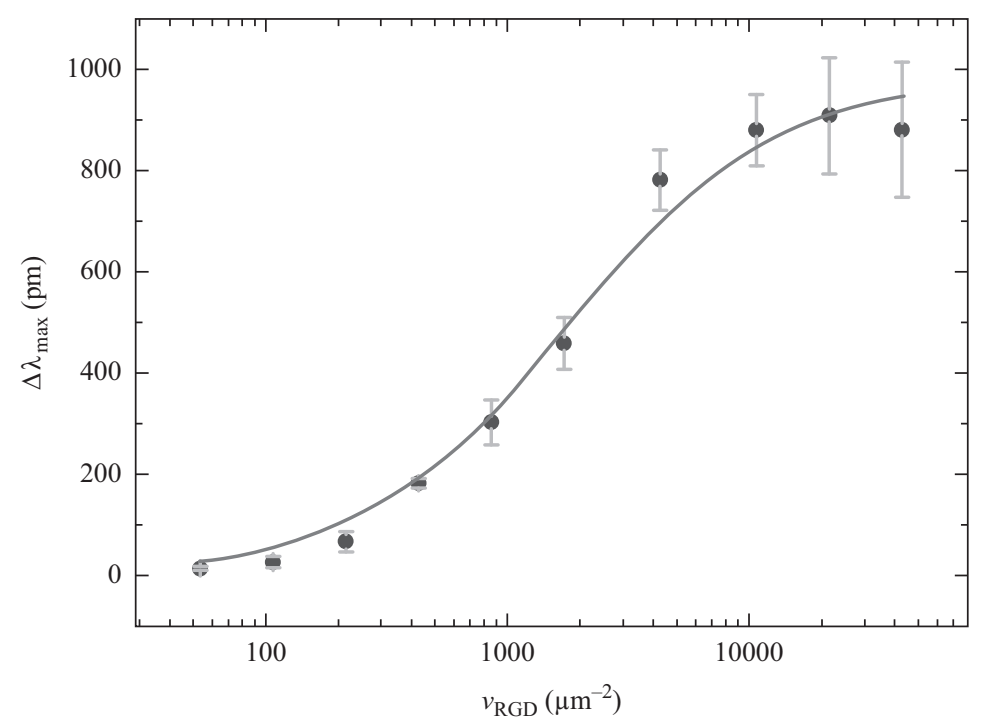

Figure 9.4 The maximum wavelength shift provoked by cells $\left(\Delta \lambda_{\max }\right)$ as a function of the surface density of RGD motifs ( $\left.v_{\mathrm{RGD}}\right)$, and the fit performed to derive the two-dimensional dissociation constant. Fitting(3)(solid line) describing the equilibrium of single-step monovalent binding to the data yielded a $2 D$ dissociation constant of ${ }^{2 D} K_{\mathrm{d}}=1753 \pm 243 \mu \mathrm{m}^{-2}$ for the binding between integrins embedded in their native (cell) membrane and the RGD motifs. Error bars on the dots represent the standard deviation from the mean. Figure is replotted from Reference 34

RGD-sequence as the RGD-specific integrins of HeLa. Second, platelet integrins isolated with a detergent and grafted uniformly into planar lipid bilayers have been claimed to be all activated, thus showing maximum affinity for their ligands [67]. In contrast, affinity regulation is an intrinsic property of $\alpha I I b \beta 3$ integrins in platelets (they are able to switch from a low affinity "inactive" to a high affinity "activated" state upon induction) [68]; thus, they are expected to show a larger dissociation constant (smaller affinity) for a certain ligand when they are in their native environment compared to when embedded into a model cell membrane system.

In summary, the simplest model described by (9.3) seems to be sufficient to characterize the integrin-ligand interaction; it fitted our data remarkably well and yielded a dissociation constant with a reasonable value.

In a recent book chapter [55], we described how the dissociation constant for the interaction between adhesion ligands and their native cell membrane receptors can be determined with the help of the Epic BT. Exceeding the limitations of most of the regular scientific articles, therein we also provide an extensive list of helpful notes and hints about the technique and all stages of the workflow; a description that will hopefully prompt and encourage future applications. 


\subsection{Outlook}

As a continuation of the experimental work described earlier and published previously (see Reference 34), we wish to further exploit the potentials of the Epic BT. Accumulating preliminary data show that cell adhesion and spreading do not always follow trivial kinetics (i.e. cannot be described by a symmetrical sigmoid). For the first time this became evident in the case of drug-treated cells. For example, varying concentrations of a small molecule adhesion inhibitor severely altered the kinetics of cell spreading, while the maximal biosensor responses were completely unaffected by it. Surprisingly, spread cell morphology showed a striking dependence on the concentration of the same drug in the assay medium, but nonetheless, the biosensor responses at saturation were the very same, i.e. they were independent of the presence of the drug [69]. In a separate study, we monitored the adhesion and spreading kinetics of unstimulated, untreated human primary monocytes, dendritic cells, and macrophages. The biosensor signal invoked by these immune cells followed nontrivial, non-monotonic kinetics [701, which was unexpected, because if untreated and unperturbed, all the other tested cell types induced a biosensor signal which could be described with a symmetrical sigmoid [29, 58].

All these findings emphasize the fundamental importance of kinetic monitoring in cell (adhesion) studies. Using more sophisticated models, kinetic data analysis may then shed light on the governing and limiting intracellular processes during cell adhesion.

\section{Acknowledgment}

The support of the Hungarian Scientific Research Fund (OTKA-PD 73084) is gratefully acknowledged. This work was supported by the Lendület program of the Hungarian Academy of Sciences, the Bolyai Scholarship, and the MedinProt grant of the Hung. Acad. Sci. to B.Szabó.

Used symbols $\lambda$ : resonant wavelength; $\Delta \lambda$ : resonant wavelength shift; $\Delta \lambda_{\text {max }}$ : maximum recorded shift in resonant wavelength; $r$ : rate constant of spreading, $m$ : constant of integration; $t$ : time; $Q$ : volume percent of the PLL-g-PEG-RGD solution in the mixed solution of copolymers; $\nu_{\mathrm{RGD}}$ : average surface density of RGD motifs; $d_{\mathrm{RGD}-\mathrm{RGD}}$ : average interligand distance; $L$ : surface concentration of unbound ligand; $R$ : surface concentration of unbound receptor; $B$ : surface concentration of the integrinligand complex; $k_{\mathrm{a}}$ : two-dimensional association rate constant; $k_{\mathrm{d}}$ : two-dimensional dissociation rate constant ${ }^{2 D} K_{\mathrm{d}}$ : $\backslash$ dimensionat dissociation constant; ${ }^{3 D} K_{\mathrm{d}}$ : threedimensional dissociation constant; $l_{\mathrm{c}}$ : confinement length.

\section{References}

[1] R. O. Hynes, "Integrins: bidirectional, allosteric signaling machines," Cell, vol. 110, pp. 673-687, 2002.

[2] R. O. Hynes, "Integrins: versatility, modulation, and signaling in cell adhesion," Cell, vol. 69, no. 1, pp. 11-25, Apr. 1992. 
[3] P. W. Wiseman, C. M. Brown, D. J. Webb, et al., "Spatial mapping of integrin interactions and dynamics during cell migration by image correlation microscopy," J. Cell Sci., vol. 117, no. Pt 23, pp. 5521-5534, Nov. 2004.

[4] A. E. Aplin, A Howe, S. K. Alahari, R. L. Juliano, and C. Hill, "Signal transduction and signal modulation by cell adhesion receptors: the role of integrins, cadherins, immunoglobulin-cell adhesion molecules, and selectins," Pharmacol. Rev., vol. 50, no. 2, pp. 198-263, 1998.

[5] C. Shi and D. I. Simon, "Integrin signals, transcription factors, and monocyte differentiation," Trends Cardiovasc. Med., vol. 16, no. 5, pp. 146-152, Jul. 2006.

[6] N. Wang, J. D. Tytell, and D. E. Ingber, "Mechanotransduction at a distance: mechanically coupling the extracellular matrix with the nucleus," Nat. Rev. Mol. Cell Biol., vol. 10, pp. 75-82, 2009.

[7] C. H. Streuli, "Integrins and cell-fate determination.," J. Cell Sci., vol. 122, no. Pt 2, pp. 171-177, Jan. 2009.

[8] D. A. Goodenough, J. A. Goliger, and D. L. Paul, "Connexins, connexons, and intercellular communication," Annu. Rev. Biochem., vol. 65, pp. 475-502, 1996.

[9] E. S. Harris, T. M. McIntyre, S. M. Prescott, and G. A. Zimmerman, "The leukocyte integrins," J. Biol. Chem., vol. 275, no. 31, pp. 23409-23412, Aug. 2000.

[10] T. A. Springer, "Adhesion receptors of the immune system," Nature, vol. 346, pp. 425-434, 1990.

[11] J. S. Desgrosellier and D. A. Cheresh, "Integrins in cancer: biological implications and therapeutic opportunities," Nat. Rev. Cancer, vol. 10, no. 1, pp. 9-22, Jan. 2010.

[12] C. Wai Wong, D. E. Dye, and D. R. Coombe, "The role of immunoglobulin superfamily cell adhesion molecules in cancer metastasis," Int. J. Cell Biol., vol. 2012, p. 340296, Jan. 2012.

[13] E. C. Boyle and B. B. Finlay, "Bacterial pathogenesis: exploiting cellular adherence," Curr. Opin. Cell Biol., vol. 15, no. 5, pp. 633-639, Oct. 2003.

[14] P. L. Stewart and G. R. Nemerow, "Cell integrins: commonly used receptors for diverse viral pathogens," Trends Microbiol., vol. 15, no. 11, pp. 500-507, Nov. 2007.

[15] E. Décavé, D. Garrivier, Y. Bréchet, B. Fourcade, and F. Bruckert, "Shear flow-induced detachment kinetics of Dictyostelium discoideum cells from solid substrate," Biophys. J., vol. 82, no. 5, pp. 2383-2395, May 2002.

[16] A. George and T. L. Proulx, "Relationship between 3 T3 cell spreading and the strength of adhesion on glass and silane surfaces," vol. 14, no. 4, 1993.

[17] E. Potthoff, O. Guillaume-Gentil, D. Ossola, et al., "Rapid and serial quantification of adhesion forces of yeast and mammalian cells," PLoS One, vol. 7, no. 12, p. e52712, Jan. 2012.

[18] G. Sagvolden, I. Giaever, E. O. Pettersen, and J. Feder, "Cell adhesion force microscopy," Proc. Natl. Acad. Sci. U. S. A., vol. 96, no. 2, pp. 471-476, Jan. 1999. 
[19] R. Salánki, C. Hős, N. Orgovan, et al., "Single cell adhesion assay using computer controlled micropipette," PLoS One, vol. 9, no. 10, p. e111450, Jan. 2014.

[20] S. S. Hur, Y. Zhao, Y-S. Li, E. Botvinick, and S. Chien, "Live cells exert 3-dimensional traction forces on their substrata," Cell. Mol. Bioeng., vol. 2, no. 3, pp. 425-436, Sep. 2009.

[21] JH-C. Wang and J-S. Lin, "Cell traction force and measurement methods," Biomech. Model. Mechanobiol., vol. 6, no. 6, pp. 361-371, Nov. 2007.

[22] D. C. Worth and M. Parsons, "Advances in imaging cell-matrix adhesions," J. Cell Sci., vol. 123, no. Pt 21, pp. 3629-3638, Nov. 2010.

[23] Y. Fang, "Label-free cell-based assays with optical biosensors in drug discovery," Assay Drug Dev. Technol., vol. 4, no. 5, pp. 583-595, 2006.

[24] J. Wegener, C. R. Keese, and I. Giaever, "Electric cell-substrate impedance sensing (ECIS) as a noninvasive means to monitor the kinetics of cell spreading to artificial surfaces," Exp. Cell Res., vol. 259, no. 1, pp. 158-166, Aug. 2000.

[25] J. M. Atienza, J. Zhu, X. Wang, X. Xu, and Y. Abassi, "Dynamic monitoring of cell adhesion and spreading on microelectronic sensor arrays," J. Biomol. Screen., vol. 10, no. 8, pp. 795-805, Dec. 2005.

[26] A. W. Peterson, M. Halter, A. Tona, K. Bhadriraju, and A. L. Plant, "Using surface plasmon resonance imaging to probe dynamic interactions between cells and extracellular matrix," Cytometry. A, vol. 77, no. 9, pp. 895-903, Sep. 2010.

[27] V. Yashunsky, V. Lirtsman, M. Golosovsky, D. Davidov, and B. Aroeti, "Real-time monitoring of epithelial cell-cell and cell-substrate interactions by infrared surface plasmon spectroscopy," Biophys. J., vol. 99, no. 12, pp. 4028-4036, Dec. 2010.

[28] K. Tiefenthaler and W. Lukosz, "Sensitivity of grating couplers as integrated-optical chemical sensors," J. Opt. Soc. Am. B, vol. 6, no. 2, p. 209, Feb. 1989.

[29] N. Orgovan, R. Salánki, N. Sándor, et al., "In-situ and label-free optical monitoring of the adhesion and spreading of primary monocytes isolated from human blood: dependence on serum concentration levels," Biosens. Bioelectron., vol. 54, pp. 339-344, Apr. 2014.

[30] R. Horvath, K. Cottier, H. C. Pedersen, and J. J. Ramsden, "Multidepth screening of living cells using optical waveguides," Biosens. Bioelectron., vol. 24, no. 4, pp. 805-810, Dec. 2008.

[31] J. J. Ramsden and R. Horvath, "Optical biosensors for cell adhesion," J. Recept. Signal Transduct. Res., vol. 29, no. 3-4, pp. 211-223, Jan. 2009.

[32] S. M. Shamah and B. T. Cunningham, "Label-free cell-based assays using photonic crystal optical biosensors," Analyst, vol. 136, no. 6, pp. 1090-1102, Mar. 2011.

[33] Y. Fang, A. M. Ferrie, N. H. Fontaine, J. Mauro, and J. Balakrishnan, "Resonant waveguide grating biosensor for living cell sensing," Biophys. J., vol. 91, no. 5, pp. 1925-1940, Sep. 2006. 
[34] N. Orgovan, B. Peter, S. Bősze, J. J. Ramsden, B. Szabó, and R. Horvath, "Dependence of cancer cell adhesion kinetics on integrin ligand surface density measured by a high-throughput label-free resonant waveguide grating biosensor," Sci. Rep., vol. 4, p. 4034, Jan. 2014.

[35] J. Comley, "Progress in the implementation of label-free detection part I: cell-based assays," Drug Discov. World Summer 2008, 2008.

[36] N. Orgovan, B. Kovacs, E. Farkas, et al., "Bulk and surface sensitivity of a resonant waveguide grating imager," Appl. Phys. Lett., vol. 104, no. 8, p. 083506 , Feb. 2014.

[37] R. Horvath, H. C. Pedersen, N. Skivesen, D. Selmeczi, and N. B. Larsen, "Monitoring of living cell attachment and spreading using reverse symmetry waveguide sensing," Appl. Phys. Lett., vol. 86, no. 7, p. 071101, 2005.

[38] V. Goral, Q. Wu, H. Sun, and Y. Fang, "Label-free optical biosensor with microfluidics for sensing ligand-directed functional selectivity on trafficking of thrombin receptor," FEBS Lett., vol. 585, no. 7, pp. 1054-1060, Apr. 2011.

[39] Y. Fang, "Label-free receptor assays," Drug Discov. Today. Technol., vol. 7, no. 1, pp. e5-e11, Jan. 2011.

[40] R. Schröder, J. Schmidt, S. Blättermann, et al., "Applying label-free dynamic mass redistribution technology to frame signaling of $\mathrm{G}$ proteincoupled receptors noninvasively in living cells," Nat. Protoc., vol. 6, no. 11, pp. 1748-1760, Nov. 2011.

[41] N. Orgovan, D. Patko, C. Hos, et al., "Sample handling in surface sensitive chemical and biological sensing: a practical review of basic fluidics and analyte transport," Adv. Colloid Interface Sci., vol. 211C, pp. 1-16, Sep. 2014.

[42] A. M. Ferrie, Q. Wu, and Y. Fang, "Resonant waveguide grating imager for live cell sensing," Appl. Phys. Lett., vol. 97, no. 22, p. 223704, Nov. 2010.

[43] Y. Fang, "Label-free biosensors for cell biology," Int. J. Electrochem., vol. 2011, pp. 1-16, 2011.

[44] J. J. Ramsden, S. Y. Li, E. Heinzle, and J. E. Prenosil, "Optical method for measurement of number and shape of attached cells in real time," Cytometry, vol. 19, no. 2, pp. 97-102, Feb. 1995.

[45] C. A. Reinhart-King, M. Dembo, and D. A. Hammer, "The dynamics and mechanics of endothelial cell spreading," Biophys. J., vol. 89, no. 1, pp. 676-689, Jul. 2005.

[46] B. J. Dubin-Thaler, G. Giannone, H-G. Döbereiner, and M. P. Sheetz, "Nanometer analysis of cell spreading on matrix-coated surfaces reveals two distinct cell states and STEPs," Biophys. J., vol. 86, no. 3, pp. 1794-1806, Mar. 2004.

[47] B. F. Bell, M. Schuler, S. Tosatti, M. Textor, Z. Schwartz, and B. D. Boyan, "Osteoblast response to titanium surfaces functionalized with extracellular matrix peptide biomimetics," Clin. Oral Implants Res., vol. 22, no. 8, pp. 865-872, Aug. 2011.

[48] S. VandeVondele, J. Vörös, and J. A. Hubbell, "RGD-grafted poly-L-lysinegraft-(polyethylene glycol) copolymers block non-specific protein adsorption 
while promoting cell adhesion," Biotechnol. Bioeng., vol. 82, no. 7, pp. 784-790, Jun. 2003.

[49] M. Schuler, G. R. Owen, D. W. Hamilton, et al., "Biomimetic modification of titanium dental implant model surfaces using the RGDSP-peptide sequence: a cell morphology study," Biomaterials, vol. 27, no. 21, pp. 4003-4015, Jul. 2006.

[50] E. Ruoslahti, "RGD and other recognition sequences for integrins," Annu. Rev. Cell Dev. Biol., vol. 12, pp. 697-715, Jan. 1996.

[51] U. Hersel, C. Dahmen, and H. Kessler, "RGD modified polymers: biomaterials for stimulated cell adhesion and beyond," Biomaterials, vol. 24, no. 24, pp. 4385-4415, Nov. 2003.

[52] J. Huang, S. V. Gra, F. Corbellini, et al., "Impact of order and disorder in RGD nanopatterns on cell adhesion," Nano Lett., vol. 9, pp. 1111-1116, 2009.

[53] E. A. Cavalcanti-Adam, T. Volberg, A. Micoulet, H. Kessler, B. Geiger, and J. P. Spatz, "Cell spreading and focal adhesion dynamics are regulated by spacing of integrin ligands," Biophys. J., vol. 92, no. 8, pp. 2964-2974, Apr. 2007.

[54] T. Frisch and O. Thoumine, "Predicting the kinetics of cell spreading," J. Biomech., vol. 35, no. 8, pp. 1137-1141, Aug. 2002.

[55] N. Orgovan, B. Peter, S. Bosze, J. J. Ramsden, B. Szabó, and R. Horvath, "Label-free profiling of cell adhesion: determination of the dissociation constant for native cell membrane adhesion receptor-ligand interaction," in Label-Free Biosensor Methods in Drug Discovery, Y. Fang, Ed. Springer, 2015, pp. 327-338.

[56] G. L. Kenausis, J. Vo, D. L. Elbert, et al., "Poly(L-lysine )-g-poly(ethylene glycol) layers on metal oxide surfaces: attachment mechanism and effects of polymer architecture on resistance to protein adsorption," pp. 3298-3309, 2000.

[57] M. Barczyk, S. Carracedo, and D. Gullberg, "Integrins," Cell Tissue Res., vol. 339, no. 1, pp. 269-280, Jan. 2010.

[58] A. Aref, R. Horvath, and J. J. Ramsden, "Spreading kinetics for quantifying cell state during stem cell differentiation," J. Biol. Phys. Chem., vol. 10, no. November, pp. 1-7, 2010.

[59] AÁ. Rauscher, Z. Simon, G. J. Szöllosi, L. Gráf, I. Derényi, and A. MalnasiCsizmadia, "Temperature dependence of internal friction in enzyme reactions," FASEB J., vol. 25, no. 8, pp. 2804-2813, Aug. 2011.

[60] M. Arnold, E. A. Cavalcanti-Adam, R. Glass, et al., "Activation of integrin function by nanopatterned adhesive interfaces," Chemphyschem, vol. 5, no. 3, pp. 383-388, Mar. 2004.

[61] G. I. Bell, M. Dembo, and P. Bongrand, "Cell adhesion. Competition between nonspecific repulsion and specific bonding," Biophys. J., vol. 45, no. 6, pp. 1051-1064, Jun. 1984.

[62] M. L. Dustin, S. K. Bromley, M. M. Davis, and C. Zhu, "Identification of self through two-dimensional chemistry and synapses," Annu. Rev. Cell Dev. Biol., vol. 17, pp. 133-157, Jan. 2001. 
178 Nanobiosensors for personalized and onsite biomedical diagnosis

[63] C. S. Izzard and L. R. Lochner, "Cell-to-substrate contacts in living fibroblasts: an interference reflexion study with an evaluation of the technique," J. Cell Sci., vol. 21, no. 1, pp. 129-159, Jun. 1976.

[64] K-F. Giebel, C. Bechinger, S. Herminghaus, et al., "Imaging of cell/ substrate contacts of living cells with surface plasmon resonance microscopy," Biophys. J., vol. 76, no. 1, pp. 509-516, Jan. 1999.

[65] C. M. Lo, M. Glogauer, M. Rossi, and J. Ferrier, "Cell-substrate separation: effect of applied force and temperature," Eur. Biophys. J., vol. 27, no. 1, pp. 9-17, Jan. 1998.

[66] M. Pfaff, K. Tangemann, B. Muller, et al., "Selective recognition of cyclic RGD peptides of NMR defined conformation by alpha(II)beta(3), alpha(V) beta(3), and alpha(5)beta(1) integrins," J. Biol. Chem., vol. 269, no. 82, pp. 20233-20238, 1994.

[67] B. Müller, H. G. Zerwes, K. Tangemann, J. Peter, and J. Engel, “Two-step binding mechanism of fibrinogen to alpha(IIb)beta(3) integrin reconstituted into planar lipid bilayers," J. Biol. Chem., vol. 268, no. 9, pp. 6800-6808, Mar. 1993.

[68] H. Ginsberg, X. De, and F. Plow, "Inside-out integrin signaling," Curr. Opin. Cell Biol., vol. 4, pp. 766-771, 1992.

[69] N. Orgován, B. Szabó, and R. Horvath, "Analysis of non-trivial cell adehesion profiles measured with a label free-optical biosensor," in press., p. 70 .

$[701$ N. Orgovan, U-S. Rita, N. Sándor, et al., "Label -free kinetic monitoring of the adhesion kinetics of human primary monocytes, dendritic cells, and macrophages on fibrinogen- and PLL-g-PEG-RGD- coated surfaces," in press. 


\section{Chapter 9 \\ Optical waveguide-based biosensor for label-free monitoring of living cells}

\footnotetext{
Author Queries

AQ1: Please confirm if "hidrophobity" is fine here.

AQ2: Please confirm if this replotting is done with permission from the Publisher of Reference 34.

AQ3: Please confirm if this replotting is done with penmIssion from the Publisher of Reference 34.

AQ4: Please confirm if this replotting Is done with permission from the Publisher of Reference 34.

AQ5: Please provide complete details of References 56, 69 and 70.
}

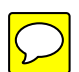

